\title{
The impact of phase state of guest histidine on properties and practical applications of nanohybrids on InSe and GaSe basis
}

\author{
F.O. IVASHCHYSHYN ${ }^{1}$, I.I. GRYGORCHAK ${ }^{1}$, O.V. BALABAN ${ }^{1}$, B.O. SEREDYUK ${ }^{2, *}$ \\ ${ }^{1}$ Lviv Polytechnic National University, 12 Bandera St., Lviv, 79013, Ukraine \\ ${ }^{2}$ National Academy of Land Forces, 32 Geroiv Majdanu St., Lviv 79012, Ukraine
}

\begin{abstract}
A new technological approach to the synthesis of multilayer nanostructures which allows their use in high-performance storage of electrical energy at the nanoscale level is discussed in this paper. In particular, the effect of co-intercalation of histidine (his), water and a solution of $\mathrm{KOH}$ into layered semiconductors of GaSe and InSe on the charge accumulation are studied. Based on the data of the cyclic current-voltage characteristics (CVC) a power storage mechanism (capacitive/pseudocapacitive) in each of these structures is described. This mechanism is in a good accord with the results of galvanostatic studies. The simulation of the parameters of the impedance equivalent circuit has been carried out, proving the possibility of using the described structures for nanoelectronics and nanoenergy devices. The observed values of tangent of electrical $\operatorname{losses} \operatorname{tg} \delta(<1)$ in coherence with a high dielectric constant $\epsilon$ are promising for the creation of quantum batteries and capacitors.
\end{abstract}

Keywords: histidine intercalation; impedance spectroscopy; negative capacitance; quantum batteries

(C) Wroclaw University of Science and Technology.

\section{Introduction}

Due to the rapid development of nanoengineering (nanoelectronics, spintronics, quantum coherence, etc.) and associated with it need to start creating highly capacitive electricity accumulators, the attention of scientists is drawn to the formation of heterostructured nonorganic-, organic- and bio-nanocomposite materials. The latter ones are believed to have unique physical and chemical properties [1,2].

The techniques, such as vacuum deposition, photolithography and Langmuir-Blodgett technique, are employed to obtain bio/nonorganicnanocomposite materials. In this respect, little experience has been gained up to date, and only first steps have been made [3, 4].

Intercalation technology provides all necessary tools to form complex atomic-molecular complexes, using a specific sequence of chemical reactions ensuring reliable anticoagulant matrix isolation of the nanosystem. That is why this

*E-mail: b.seredyuk@gmail.com technology was chosen by us to synthesize multilayered structures with semiconductor (InSe) and molecular nematic nanolayers [5]. The obtained nanohybrids can be used as high-capacity variconds and nanostructured elements of delay lines which can be integrated directly into nanochips.

Structures, with alternate semiconductor and nematic nanolayers, have been thoroughly studied in the literature [6], so this work is an attempt to find new ways to synthesize multilayer nanostructures involving organic and bio-nanocomposite materials. Furthermore, up to date, quite little is known about the bioorganic/inorganic-semiconductor multilayered nanohybrids. Therefore, the aim of this work is to provide a new insight into this field of research.

\section{Experimental}

With a purpose to form multilayered nanohybrid structures by means of intercalation, single crystals, such as gallium selenide (GaSe) and indium selenide (InSe), were used as a "host" material. They are characterized by a layered structure, 
and $\mathrm{p}$ or n-type conductivity. These single crystals have a high photosensitivity in the visible and near infrared region, and the band gap (according to the optical data) of $2.02 \mathrm{eV}$ for the first type of single crystals, and $1.22 \mathrm{eV}$ for the other one. These structures have different types of chemical bonds: along the layers - strong covalent, between the layers - weak van der Waals that causes strong anisotropy of single crystal properties along and across the layers. It is known [7], that GaSe and InSe have "guest" positions due to weak van der Waals forces oriented perpendicular to the crystallographic c axis. The "guest" components are intercalated into these very positions. The amino acid histidine $\left(\mathrm{C}_{6} \mathrm{H}_{9} \mathrm{~N}_{3} \mathrm{O}_{2}\right) \bullet$ (his) was chosen as the "guest" one as in the isoelectric point $(\mathrm{pH}$ at which the net charge of amino acid molecule is equal to zero), it is a zwitterion possessing the properties of both anion and cation with a huge dipole moment.

Formation of the above mentioned multilayered nanohybrid structures was carried out by a three-stage crystal engineering scheme described in detail in the literature [5]. As a result, sample configurations of $\mathrm{GaSe}$ his > and $\mathrm{InSe}$ 〈his〉 have been obtained, where the expansion of the semiconductor matrix was of a threefold value. Heteroaggregate nanohybrids of $\mathrm{GaSe}\left\langle\right.$ his $+\mathrm{H}_{2} \mathrm{O}$ 〉 and InSe $\left\langle\right.$ his $\left.+\mathrm{H}_{2} \mathrm{O}\right\rangle$ were obtained by the transition of histidine into the liquid phase which, in turn, was performed by co-intercalation from the distilled water. Additional co-intercalation by $30 \%$ aqueous $\mathrm{KOH}$ solution was carried out in order to change the $\mathrm{pH}$ value. As a result, the multilayered nanohybrids of $\mathrm{GaSe}\left\langle\right.$ his $\left.+\mathrm{H}_{2} \mathrm{O}+\mathrm{KOH}\right\rangle$ and $\mathrm{InSe}$ his $\left.+\mathrm{H}_{2} \mathrm{O}+\mathrm{KOH}\right\rangle$ were formed.

Studies of kinetic and polarization properties of the synthesized nanostructures were carried out at room temperature perpendicular and alongside the layers of GaSe and InSe single crystals by impedance spectroscopy in the frequency range of $10^{-3} \mathrm{~Hz}$ to $10^{6} \mathrm{~Hz}$, with a measuring complex "AUTOLAB" by the Company "ECO CHEMIE" (Netherlands), and using a computer programs FRA-2 and GPES. Computer parametric identification of the acquired impedance dependences has been conducted within ZView 2.3 (Scribner
Associates) programming package. Cyclic voltammetry (CVC) was recorded at a rate of sweep voltage of $0.05 \mathrm{~V} / \mathrm{s}$. Cycles "charge-discharge" were carried out in galvanostatic mode using the "AUTOLAB" measuring complex.

\section{Results and discussion}

Impedance data of the original expanded GaSe matrix (curve 1 in Fig. 1) measured perpendicular to the layer showed a usual situation: the corresponding impedance hodograph has a two arc character and reflects capacitive feedback of the localized states and the frequency dependent impedance. This impedance is caused by hopping between localized states near the Fermi level in the system of atomic monolayers: high frequency arc (HF). Midrange arc (MF) reflects the currents through the intervals sustained by van der Waals forces. These arcs are modeled by a finite element of a constant phase (BCPE) [8] which emulates the current flow in the space-limited area with a complex electrical conductivity [9]. Most lowfrequency (LF) section reflects the distribution of the active resistance element (due to discrete energy spectrum of the extended GaSe matrix) which is modeled by a constant phase element (CPE) with low phase deviation $\xi<0.1$ [9]. A respective electrical circuit in this case is shown in Fig. 1a. Introduction of histidine leads to an increase in the frequency dispersion of the impedance locus and points to the emergence of other potential barriers. The equivalent circuit diagram in this case is substantially altered (Fig. 1b). In particular, it has received two units, where the latter one contains the element of inductance, the presence of which is due to the transition of low-frequency branch of the Nyquist diagram into IV inductive quadrant of the plane of the complex impedance.

Parameters of the model (a) and (b) are listed

Table 1. Parameters of the model (a).

\begin{tabular}{lcccc}
\hline $\begin{array}{l}\text { Element } \\
\text { of the model }\end{array}$ & $\mathrm{BCPE}_{1}$ & $\mathrm{BCPE}_{2}$ & $\mathrm{R}$ & $\mathrm{CPE}$ \\
{$[\Omega]$} & {$[\Omega]$} & {$[\mathrm{F}]$} \\
\hline \hline Value & $2.15 \mathrm{E} 8$ & $1.01 \mathrm{E} 8$ & $1.73 \mathrm{E} 8$ & $4.47 \mathrm{E}-12$ \\
\hline
\end{tabular}




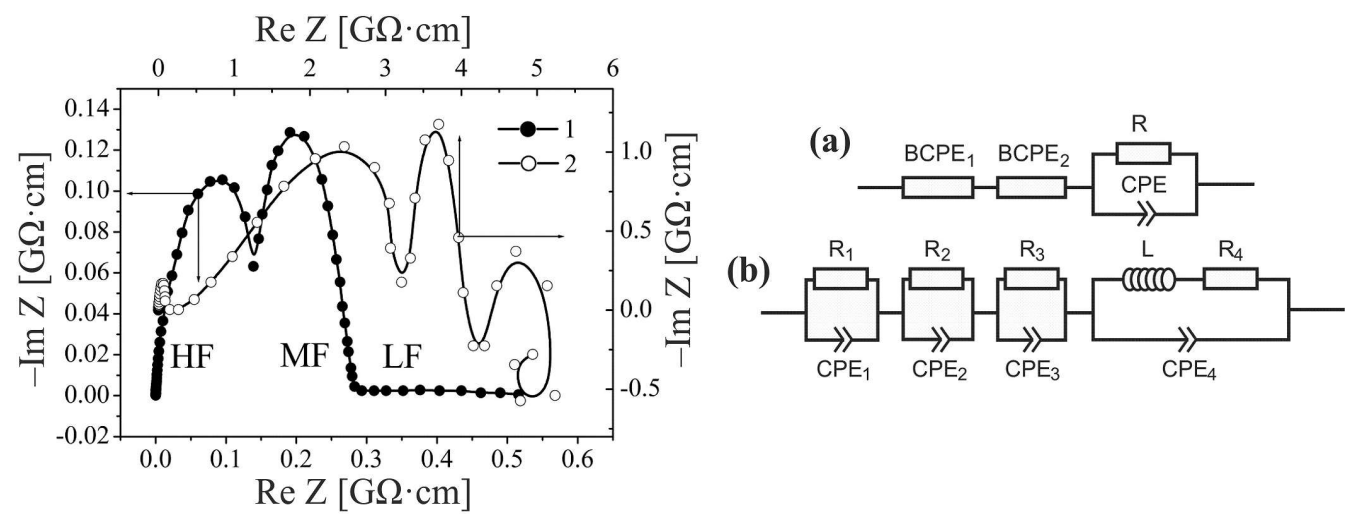

Fig. 1. Nyquist diagrams of the original expanded GaSe matrix (1), and nanostructure of GaSe 〈his〉(2) with the respective equivalent circuit diagrams [10]. Equivalent circuit diagrams are shown in the insets (a) and (b) on the righthand side of the figure.

Table 2. Parameters of the model (b).

\begin{tabular}{lccccccccc}
\hline $\begin{array}{l}\text { Element } \\
\text { of the model }\end{array}$ & $\begin{array}{c}\mathrm{R}_{1} \\
{[\Omega]}\end{array}$ & $\begin{array}{c}\mathrm{CPE}_{1} \\
{[\mathrm{~F}]}\end{array}$ & $\begin{array}{c}\mathrm{R}_{2} \\
{[\Omega]}\end{array}$ & $\begin{array}{c}\mathrm{CPE}_{2} \\
{[\mathrm{~F}]}\end{array}$ & $\begin{array}{c}\mathrm{R}_{3} \\
{[\Omega]}\end{array}$ & $\begin{array}{c}\mathrm{CPE}_{3} \\
{[\mathrm{~F}]}\end{array}$ & $\begin{array}{c}\mathrm{L} \\
{[\mathrm{H}]}\end{array}$ & $\begin{array}{c}\mathrm{R}_{4} \\
{[\Omega]}\end{array}$ & $\begin{array}{c}\mathrm{CPE}_{4} \\
{[\mathrm{~F}]}\end{array}$ \\
\hline \hline Value & $6.57 \mathrm{E} 7$ & $2.93 \mathrm{E}-14$ & $2.96 \mathrm{E} 8$ & $4.79 \mathrm{E}-12$ & $8.77 \mathrm{E} 8$ & $1.73 \mathrm{E}-8$ & $4.63 \mathrm{E} 3$ & $3.04 \mathrm{E} 9$ & $1.17 \mathrm{E}-10$ \\
\hline
\end{tabular}

in Table 1 and Table 2, respectively. This behavior of the impedance locus is known as the phenomenon of "negative" capacity, the mechanism which has not been yet fully understood $[11,12]$. This phenomenon is promising for the development of nanoscale delay lines without gyrators.

Another interesting feature of the synthesized nanostructures with internal inductance is their ability to generate low-frequency pulses, as shown in Fig. 2.

Transition of histidine into the liquid phase and the change in $\mathrm{pH}$ alters the form of the impedance locus (Fig. 3a) measured perpendicular to the nanohybrid layers. In this case, the observed mid-frequency inductive response has obviously a different nature than for the solid phase guest, what might be caused by a combination of the relaxations due to charge transfer and charge accumulation [13].

The latter one, in turn, can show the charge accumulation on the molecular level at the interface maintaining the operation of these nanoaccumulator single crystals. The results of CVC and galvanostatic charge-discharge cycles, shown in Fig. 3b, might be a proof of this. Here, a pseudocapacitive nature of the charge accumulation in the structure of $\mathrm{GaSe}\left\langle\right.$ his $\left.+\mathrm{H}_{2} \mathrm{O}+\mathrm{KOH}\right\rangle$ and the corresponding curve of the direct current charge-discharge (inset in Fig. 3b) are clearly seen. The asymmetry of the charge-discharge curves indicates the spontaneous generation of electromotive force (EMF), whose mechanism is not understood yet. The reason for that may be as follows: synthesized nanohybrids represent multilayer heterophase structure of alternating semiconductor and quasi-two-dimensional ionic layers with existing shallow acceptor levels having ionization energies $E_{i}$ ranging from $0.015 \mathrm{eV}$ to $0.025 \mathrm{eV}$ as well as amino acidic multi-oxidation recovery centers. In such structures, endothermic spontaneous generation of EMF in the direction perpendicular to nanolayers occurs, when at a certain temperature the effective Bohr radius (with energy $E_{i}$ ) matches the Debay electrostatic potential shielding radius of this defect. Thus, the generated EMF may be caused by the carriers delocalized from their native defect levels leading to a diffusion current through the interface of nanolayers with different electric charge. 

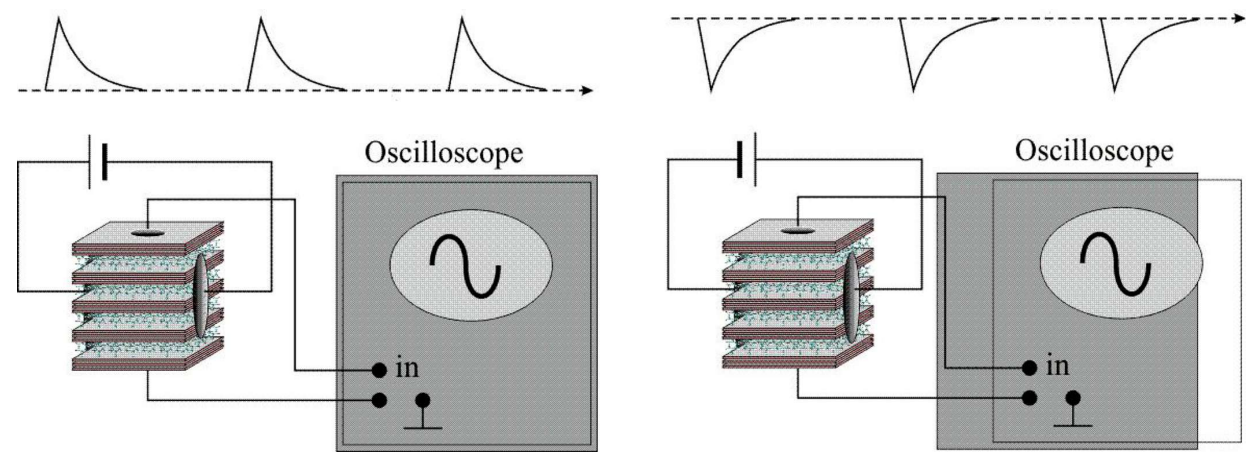

Fig. 2. Pulse generation in bio/nonorganic N-barrier GaSe 〈his〉 nanostructure.
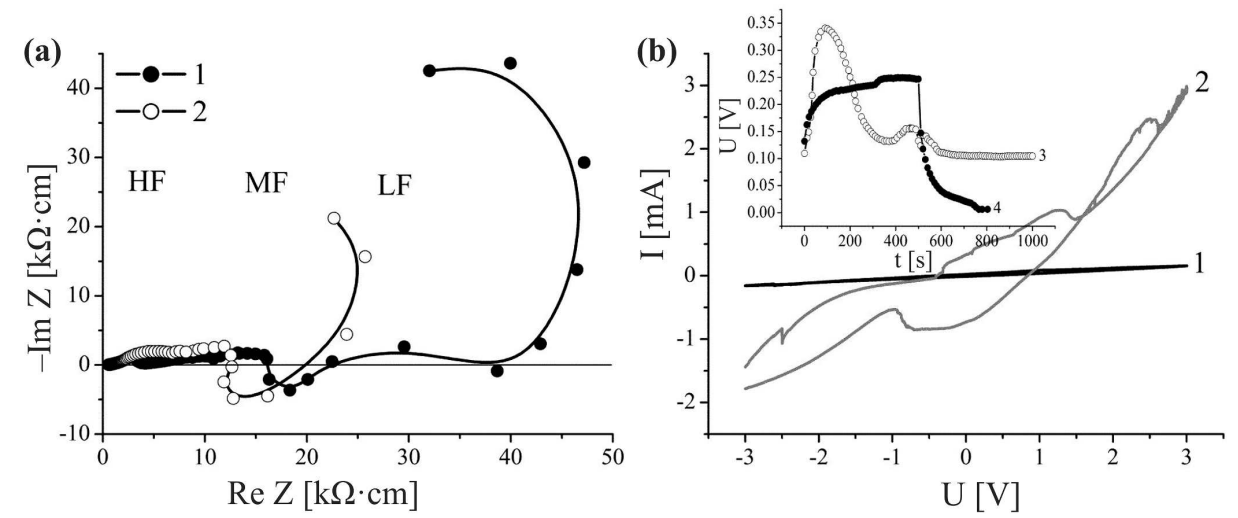

Fig. 3. Nyquist diagrams (a) and CVC (b), measured perpendicular to the nanolayers of GaSe $\left\langle\right.$ his $+\mathrm{H}_{2} \mathrm{O}$ > (1) and $\mathrm{GaSe}$ his $+\mathrm{H}_{2} \mathrm{O}+\mathrm{KOH}$ (2). Galvanostatic charge-discharge cycles of $\mathrm{GaSe}$ 〈his $+\mathrm{H}_{2} \mathrm{O}+\mathrm{KOH}$ ? the current of $1 \mu \mathrm{A}(3)$ and $10 \mu \mathrm{A}(4)$ are shown in the inset.

Nyquist diagrams of nanohybrids based on GaSe and CVC, measured along the layers are shown in Fig. 4. It is seen that the low frequency branch of the Nyquist diagram (Fig. 4a) for $\mathrm{GaSe}\left\langle\right.$ his $\left.+\mathrm{H}_{2} \mathrm{O}\right\rangle$ and $\mathrm{GaSe}$ 〈his $+\mathrm{KOH}+\mathrm{H}_{2} \mathrm{O}$ 〉 nanohybrids along the layers have opposite frequency data origin as compared to the data perpendicular to the layers (Fig. 3a).

Results of CVC (Fig. 4b) for GaSe 〈his $+\mathrm{H}_{2} \mathrm{O}+\mathrm{KOH}>$ show higher currents than the ones for $\mathrm{GaSe}$ 〈his $+\mathrm{H}_{2} \mathrm{O}$ > which is similar to the behavior of the curves measured perpendicular to the layers. Again, pseudocapacitive charge storage mechanism in this nanohybrid is clearly seen. It is expected that the currents along the layers of $\mathrm{GaSe}\left\langle\right.$ his $+\mathrm{H}_{2} \mathrm{O}$ 〉 nanostructures will be higher than in the one perpendicular to them (Fig. 3b).
The formation of histidine nanolayers in equidistantly expanded InSe matrix leads to an increase in the real part of the complex specific impedance by a factor of 40 (Fig. 5). The equivalent circuit diagram for the expanded matrix as well as for InSe 〈his〉 nanohybrids can be obtained from the impedance model shown in Fig. 1, and for GaSe by withdrawal of one of the BCPE elements. Incompleteness of arc locus of the impedance in the high frequency region is solely due to the fact that the complex impedance is limited to $1 \mathrm{MHz}$ of the scanning frequency range.

Transition of histidine into a liquid phase by slightly increasing $\operatorname{Re}(Z)$ (when measured perpendicular to the nanolayers) in most of lowfrequency areas leads to the complete transformation of its frequency dispersion, such as non-monotonous change of $\operatorname{Re}(\mathrm{Z})$ in frequency 


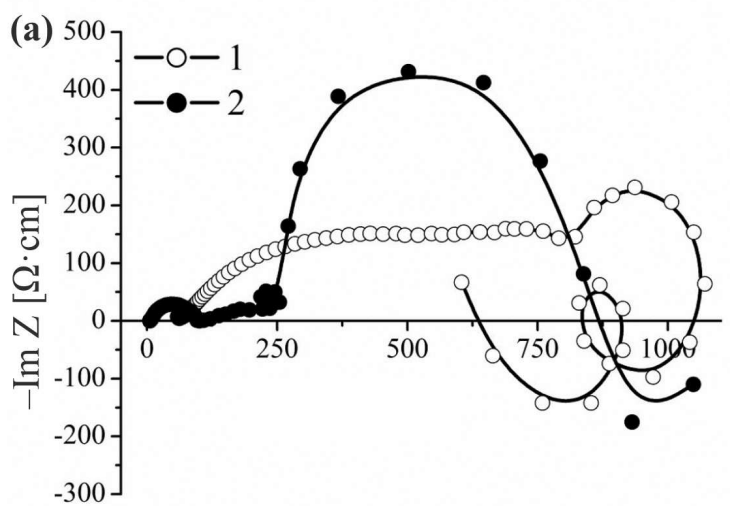

$\operatorname{Re} \mathrm{Z}[\Omega \cdot \mathrm{cm}]$

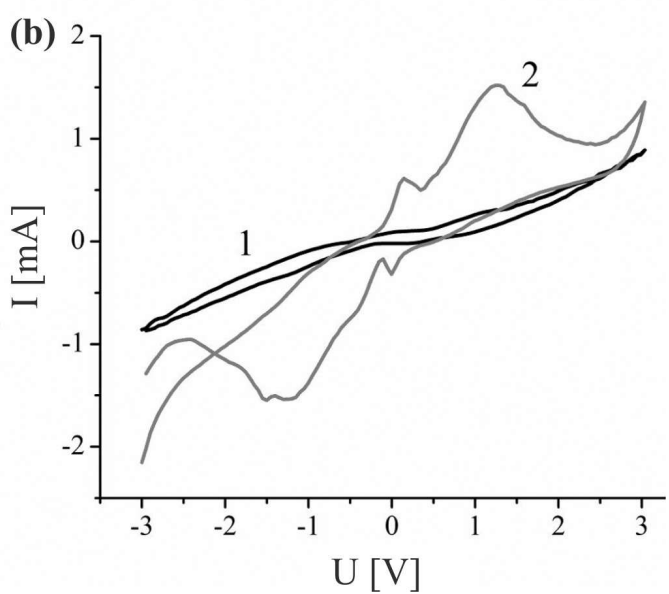

Fig. 4. Nyquist diagrams (a) and CVC (b), measured along the nanolayers of GaSe $\left\langle\right.$ his $+\mathrm{H}_{2} \mathrm{O}$ > (1) and GaSe his $+\mathrm{H}_{2} \mathrm{O}+\mathrm{KOH}$ (2).

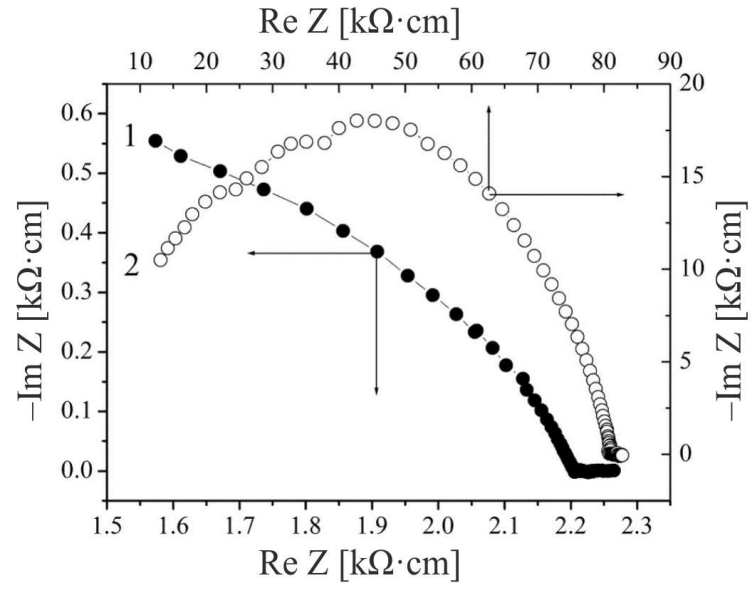

Fig. 5. Nyquist diagrams of the original expanded GaSe matrix (1) and GaSe 〈his〉 nanostructure (2).

range of $0.0015 \mathrm{~Hz}$ to $6.5 \mathrm{~Hz}$ (20-fold peak at around $0.02 \mathrm{~Hz}$ ) and a further decrease with increasing frequency. Changing $\mathrm{pH}$ of histidine leads to the significant decrease in $\operatorname{Re}(\mathrm{Z})$ over the entire frequency range as well as to the ambiguity of functional $-\operatorname{Im}(\mathrm{Z})=\mathrm{f}(\operatorname{Re}(\mathrm{Z}))$ dependence. In both cases, the respective branches of the Nyquist diagrams (Fig. 6a) show the processes of accumulation and holding the charge in the synthesized Nbarrier structures.

However, as it can be observed from the CVC data, the mechanisms of accumulation and holding the charge in general are different (Fig. 6b).
If, in case of InSe $\left\langle\right.$ his $+\mathrm{H}_{2} \mathrm{O}$, it is likely to be due to electron capture and holding the carrier in the trapping centers, then in the structure of InSe $\left\langle\right.$ his $+\mathrm{H}_{2} \mathrm{O}+\mathrm{KOH}$ > there are sufficient grounds to indicate Faraday pseudocapacitive accumulation of charge at the phase boundaries as the main mechanism of accumulation and holding the charge. The galvanostatic charge-discharge curves might be a proof of the latter one (inset in Fig. 6b) which demonstrates the formation of a single-crystal nanostructured storage of electrical energy. Moreover, in this case, the combination of ultra-high values of $\epsilon$ and low (less than 1) value of $\operatorname{tg} \delta$ (inset in Fig. 6b) in infra-low frequency area may be indicative of the quantum nature of power storage in $\mathrm{InSe}\left\langle\right.$ his $\left.+\mathrm{H}_{2} \mathrm{O}+\mathrm{KOH}\right\rangle$ (perpendicular to the layers), thus, the possibility of the formation of quantum accumulators of electrical energy $[11,12]$. For InSe $\left\langle\right.$ his $+\mathrm{H}_{2} \mathrm{O}$, in turn, similar combination of $\epsilon$ and $\operatorname{tg} \delta$ values is observed in the range of $1 \mathrm{~Hz}$ to $120 \mathrm{~Hz}$, what is promising for the development of quantum capacitors for radio frequency.

CVC measurement of our synthesized hybrids performed along the nanolayers (Fig. 7) gave the current value for InSe $<$ his $+\mathrm{KOH}+\mathrm{H}_{2} \mathrm{O}$ > smaller than for InSe $\left\langle\right.$ his $+\mathrm{H}_{2} \mathrm{O}$ > as opposed to the CVC measurement data perpendicular to the layers. According to the Nyquist diagrams, the resistance 

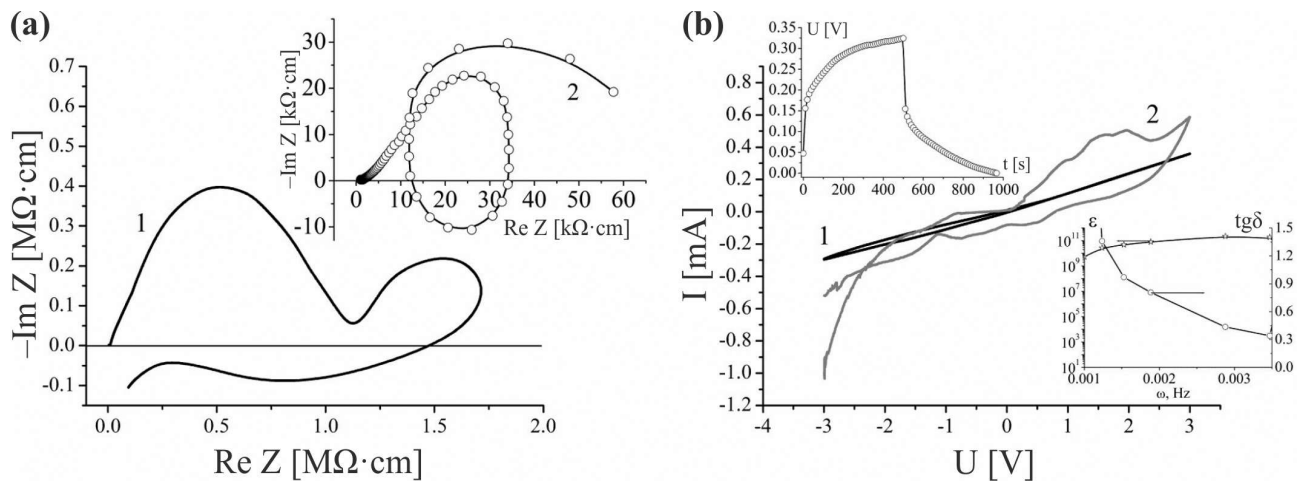

Fig. 6. Nyquist diagrams (a) and CVC (b), measured perpendicular to the nanolayers of InSe $\left\langle\right.$ his $+\mathrm{H}_{2} \mathrm{O}$ > (1) and InSe his $+\mathrm{H}_{2} \mathrm{O}+\mathrm{KOH}>$ (2). Galvanostatic charge-discharge cycle at $1 \mu \mathrm{A}$ current (top left corner), tangent of loss angle and dielectric constant (bottom right corner) of InSe $\left\langle\right.$ his $\left.+\mathrm{H}_{2} \mathrm{O}+\mathrm{KOH}\right\rangle$ are shown in the insets to Fig. $6 \mathrm{~b}$.
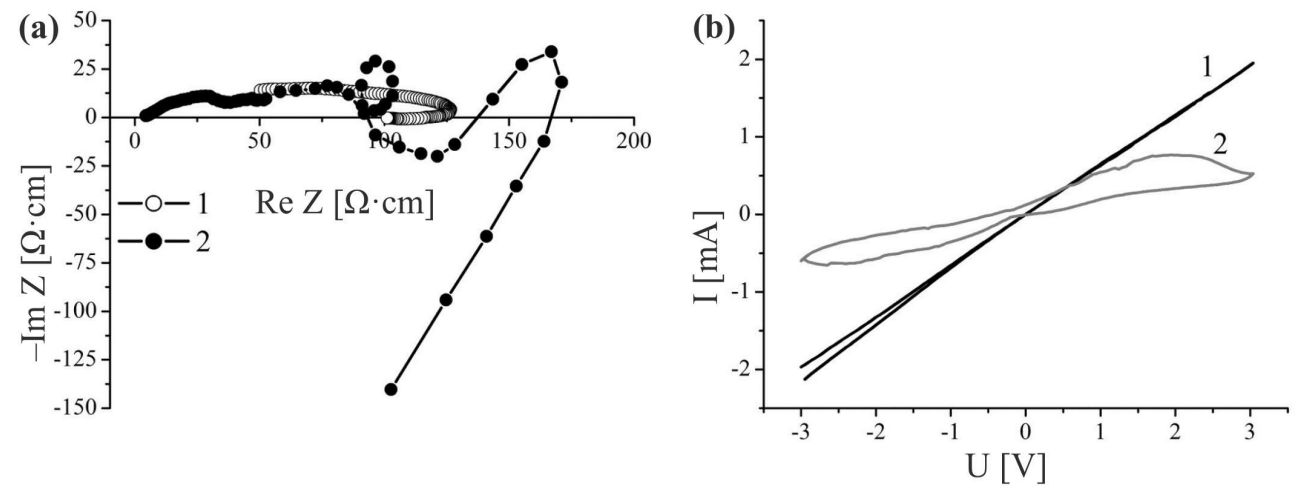

Fig. 7. Nyquist diagrams (a) and CVC (b), measured along the nanolayers for $\mathrm{InSe}\left\langle\right.$ his $+\mathrm{H}_{2} \mathrm{O}$ > (1) and $\mathrm{InSe}$ his $\left.+\mathrm{H}_{2} \mathrm{O}+\mathrm{KOH}\right\rangle(2)$.

has the tendency to decrease with increasing frequency; however, it is clearly seen only for the capacity of $\mathrm{InSe}\left\langle\right.$ his $\left.+\mathrm{H}_{2} \mathrm{O}+\mathrm{KOH}\right\rangle$ measured along the layers. On the other hand, if the resistance is measured perpendicularly to the nanolayers, InSe 〈his $+\mathrm{H}_{2} \mathrm{O}$ 〉 is subjected to the same phenomenon.

\section{Conclusions}

1. Introduction of histidine into GaSe is accompanied by the phenomenon of "negative" capacity that can be practically used to create nanoscale delay lines without using gyrators.

2. GaSe 〈his〉 nanohybrid structure can generate low-frequency pulses which may be caused by its internal inductance.
3. $\mathrm{GaSe}\left\langle\right.$ his $+\mathrm{H}_{2} \mathrm{O}+\mathrm{KOH}$ > is characterized by pseudocapacitive nature of the charge accumulation which stands for the creation of a singlecrystal nanostructured storage of electrical energy.

4. The combination of the high value of the dielectric constant $\epsilon$ and the low (less than 1) value of $\operatorname{tg} \delta$ for $\mathrm{InSe}\left\langle\right.$ his $+\mathrm{H}_{2} \mathrm{O}+\mathrm{KOH}$ (perpendicular to the layers) in the infra-low frequency range indicates the possibility of the formation of quantum accumulator of electrical energy. For InSe his $+\mathrm{H}_{2} \mathrm{O}$ 〉 this combination is observed in the range of $1 \mathrm{~Hz}$ to $125 \mathrm{~Hz}$ which is promising for the development of quantum capacitors for radio frequency. 


\section{References}

[1] Choy J.H., KWonS.J., PARK G.S., Science, 280 (1998), 1589.

[2] Choy J.H., Kwak S.Y., Park J.S., JeOng Y.J., J. Am. Chem. Soc., 121 (1999), 1399.

[3] Grygorchak I.I., Seredyuk B.O., Tovstyuk K.D., BАКнмаTYUK B.P., High-Frequency Capacitor Nanostructure Formation by Intercalation, in: Julien C., Pereira-Ramos J.P., Momchilov A. (Eds.), New Trends in Intercalation Compounds for Energy Storage. Nato Science Series II, Springer, Netherlands, 2002, p. 543.

[4] Voitovych S.A., GrygorchaK I.I., AKsimenTYEVA O.I., Mol. Cryst. Liq. Cryst., 497 (2008), 55.

[5] IVAshchyshyn F.O., Grygorchak I.I., Phys. Status Solidi A, 52 (2010), 1891.

[6] IVAShChyShyn F.O., GRYGORChAK I.I., MiKITYUK Z.M., Fechan A.V., KuliK Y.O., Russ. Phys. J.+, 53 (2010), 155.

[7] Kuhn A., Chevy A., Chevalier R., Phys. Status Solidi A, 31 (1975), 469.
[8] Stojnov Z.B., Grafov B.M., Savvova-Stojnova B.S., YelKIN V.V., Electrochemical impedance, Nauka Publishers, Moscow, 1991.

[9] Macdonald J.R., Impedance Spectroscopy, J. Wiley \& Sons, New York, 1987.

[10] IVASHCHYSHYN F.O., GRYGORCHAK I.I., KLAPCHUK M.I., SPQEO, 18 (2015), 362.

[11] Bishchaniuk T.M., Balaban O.V., Shvets R.Y., GrygorchaK I.I., Fechan A.V., Lukiyanets B.A., Ivashchyshyn F.O., Mol. Cryst. Liq. Cryst., 589 (2014), 132.

[12] GRYGORCHAK I.I., LUKIYANETS B.A., BALABAN O.V., Bishchaniuk T.M., ShVETS R.Y., Mat. Sci. Semicon. Proc., 26 (2014), 690.

[13] Bisquert J., Randriamahazaka H., GarciaBelmonte G., Electrochim. Acta, 51 (2005), 627.

Received 2016-06-22

Accepted 2016-12-23 